https://doi.org/10.15407/dopovidi2020.03.010

UDC 519.62

\author{
V.A. Prusov ${ }^{1}$, A.Yu. Doroshenko ${ }^{2}$ \\ ${ }^{1}$ Ukrainian Hydrometeorological Institute of the SSE of Ukraine and NAS of Ukraine, Kyiv \\ ${ }^{2}$ Institute of Software Systems of the NAS of Ukraine, Kyiv \\ E-mail: doroshenkoanatoliy2@gmail.com
}

\title{
An efficient computational method for mesoscale weather forecasting
}

Presented by Academician of the NAS of Ukraine P.I. Andon

Mathematical problems associated with the numerical solution of equations for the predictive models of regional atmospheric circulation are considered. A methodology is considered for effective regional solutions of boundary-value problems with a prehistory on the basis of the "one-way interaction" approach. Within this approach, a method is proposed for filling the data given on the macroscale grid nodes in the mesoscale network based on the spline interpolation and a precise (the fourth-order of accuracy) numerical method for the approximation of the first- and second-order derivatives in differential equations. Thereby, solving problems for ordinary differential equations can be carried out effectively by the interpolation.

Keywords: mesoscale weather forecasting, differential equations, interpolation.

1. Introduction. Regular grids with even steps of the spatial coordinates in the whole computational domain are the most convenient for implementing numerical methods for the integration of equations of weather forecasts. However, computing a local numerical weather forecast based on the global general circulation models of the atmosphere will need an enormous increase in computation time exceeding reasonable limits. Moreover, as some regional weather details are well localized, it is reasonable to apply high-resolution grids locally.

Separate modeling of regional atmospheric processes, in which the boundary conditions are considered unchanged for the time interval calculation, for years was only of scientific interest (e.g., see [1, 2]), since the stated lateral boundary conditions lead to large errors and even to false numerical solutions. So, to reduce the forecast errors in the restricted area, one must add a buffer zone to expand the boundaries of the area to such an extent that the generated disturbance at the boundaries does not reach the computational domain. But increasing the area of solutions entails increasing the computer performance requirements and may lead to difficulties in the practical realization of such models.

Цитування: Prusov V.A., Doroshenko A.Yu. An efficient computational method for mesoscale weather forecasting. Допов. Наи. акад. наук. Укр. 2020. № 3. С. 10-18. https://doi.org/10.15407/dopovidi2020.03.010 
In the recent years, the regional modeling of atmospheric processes is implemented in view of the fact that the field of meteorological variables in a bounded domain is formed under the influence of a macroscale atmospheric circulation. Therefore, a bounded domain of solutions is seen as a part of the whole, and the non-stationary boundary conditions at its lateral boundaries are formulated on the basis of data obtained from a bordering region. Thus, in practice, when solving the numerical weather prediction problem in a limited area, they make the grid more condensed to achieve the desired accuracy for solving the problem in the field of large gradients of dependent functions. Particularly, in [3, 4], the authors analyzed the problems arising from the application of non-uniform grids to difference schemes of numerical integration of the equations of hydrodynamics and heat and mass transfer within a regional weather prediction model. So, the combined model consists of:

1) the global atmospheric general circulation model including simplified equations numerically implemented on the coarse grid;

2) the regional model, which includes the complete equations of fluid flow, heat, and mass transfer numerically implemented on a fine grid.

The boundary conditions necessary for the regional model are identified with the solution to the global model that can be integrated together with the regional model, or in advance. This method of forecasting for the nested grids method was called the method of "one-way interaction" [5], because the numerical results of the internal model do not affect the integration of the equations of the external model.

2. The Method of "One-Way Interaction”. In the analysis of the essence of the method of "one-way interaction", it may seem that, from a computational point of view, there is no upper limit on the term of the regional forecast on embedded grids. Therefore, it was considered that the regional model containing the complete equations of fluid flow, heat, and mass transfer solved numerically on a fine grid will be the basis of operational weather prediction schemes in the near future. But in practice, non-stationary boundary conditions are determined by the extrapolation in the models of "one-way interaction". This leads to the emergence of errors spread into the area of solutions with the speed of external gravitational waves, which reduces significantly possible terms of a weather forecast for a limited area. Consequently, in order to make the regional weather forecasts real, it is necessary to continue the study of the interaction of grids with different resolutions.

The issue of a correct formulation of boundary conditions in the case of the method of "one-way interaction" is difficult enough. In the books devoted to the study of the effect of different types of boundary conditions on numerical simulation results for non-uniform grids (see, e.g., [6]), it was shown that the optimal mathematical and computational boundary conditions for the scheme with the "one-way interaction" should have the following properties:

boundary conditions must specify the main quasigeostrophic part of a solution, when the movement is directed into the region; the outlet of the solutions region should contain no reflection in the form of a computing mode or gravitational waves;

fast gravitational waves generated inside the area should, if possible, go across the border or should not be reflected and reinforced by the boundary conditions.

Most of the boundary conditions applied in practice do not meet not only both requirements, but each of them separately. 
Since the traditional numerical solution of the problem of regional weather forecasting in the formulation of classical initial-boundary-value problem is associated with a lot of difficulties discussed above, we replace it with a similar problem satisfying the special requirements arising from the concept of "one-way interaction". This may be explained with a simple example.

Let we need to find a function $\Re(t)$ at the interval $t_{j-1} \leqslant t \leqslant t_{j}$ satisfying the differential equation

$$
\frac{d \Re}{d t}=F(t)
$$

and additional conditions from the prehistory

$$
\Re\left(t_{j}\right)=\Re_{j}, \quad j=1,2, \ldots, N,
$$

where $\Re_{j}$ are the discrete values of the function $\Re(t)$ given at the nodes $t=t_{j}(j=1,2, \ldots, N)$ of the large-scale grid $\varpi_{h}$ with the grid step pitch $h_{j}=t_{j}-t_{j-1}$. In general, $F$ is a nonlinear operator. It is algebraic for ordinary differential equations and is spatial differential for partial differential equations.

At first, we restrict ourselves to problem (1) - (2) in the case of an ordinary differential equation. The natural way to solve this problem is as follows. On the segment $\left[t_{j-1}, t_{j}\right]$, we introduce a small-scale grid $\Phi_{h}$ of points $\zeta_{k}(k=0,1, \ldots, M)$ with the increment of $\tau_{k-1}=\zeta_{k}-\zeta_{k-1}$ provided that $\zeta_{0}=t_{j-1}, \zeta_{M}=t_{j}$ and consider the differential equation not everywhere on $t$, but only on the interval $\left[t_{j-1}, t_{j}\right]$. Replacing the derivative $d \Re\left(\zeta_{k}\right) / d t$ by its approximate value, for example, $d \Re\left(\zeta_{k}\right) / d t=\left[\Re\left(\zeta_{k+1}\right)-\Re\left(\zeta_{k}\right)\right] / \tau_{k} \equiv\left(\Re_{k+1}-\Re_{k}\right) / \tau_{k}$, we move to the difference scheme

$$
\Re_{k+1}=\Re_{k}+\tau_{k} F\left(\zeta_{k}\right), \quad k=0,1, \ldots, M-1 .
$$

The initial value of $\mathfrak{R}\left(\zeta_{0}\right)=\mathfrak{R}\left(t_{j-1}\right)=\Re_{j-1}$ is defined. Other successive values $\mathfrak{R}\left(\zeta_{0}\right)=$ $=\Re\left(t_{j-1}\right)=\Re_{j-1}$ are found by solving the differential equation (3). That is, to solve the problem (1), (2), one can apply a difference scheme for the Cauchy problem.

It can be done also in a different way [4]. Taking a closer look at the statement of the problem (1), (2), one can see that finding the value of $\Re(t)$ in the interval $\left[t_{j-1}, t_{j}\right]$ is a task of interpolation. Indeed, at the points $t=t_{j}(j=1,2, \ldots, N)$, we know the values of the function $\Re_{j}=\Re\left(t_{j}\right)$ and values of the right side of Eq. (1) $F\left(t_{j}\right)$, i.e., its first derivative $d \mathfrak{R} /\left.d t\right|_{t=t_{j}}=F\left(t_{j}\right)$. Under the terms of problem (1), (2), one must find $\mathfrak{R}(t)$ for the value of $t$ different from the values of the nodes $t=t_{j}(j=1,2, \ldots, N)$. Hence, we come to the method known as the interpolation "with multiple nodes" or Hermite polynomial interpolation.

3. A numerical method based on a prehistory and interpolation with multiple nodes for solving the non-stationary problem. The following will consider the general problem of interpolation functions by a set of its several values (prehistory) and a derivative of the first order with the help of an algebraic polynomial. An appropriate representation of the polynomial interpolation will be obtained, its uniqueness proved, and interpolation error estimated.

Suppose that in addition to the values of the function $\mathfrak{R}_{j}$, its first order derivatives $\Re_{j}^{\prime}$ are given at the nodes of the grid under consideration so that the total of known data is $n+1=2 N$. Suppose, furthermore, the values of $t$ does not coincide with the grid points $t_{j}(j=1,2, \ldots, N)$, 
where the values $\Re_{j}^{(a)}, \alpha=0,1$ are given. It is required, using the source data, to find a value $\Re(t)$, i.e. to solve the problem of interpolation. To do this, one must specify a rule by which, based on given values $t_{j}$ and $\Re^{(a)}\left(t_{j}\right), \alpha=0,1,(j=1,2, \ldots, N)$, we can calculate the approximate value of $\Re(t)$.

It is known that if the function $\Re$ on the interval $a \leqslant t \leqslant b$ of small length has derivatives of a sufficiently high order, then their behavior on a small portion of it is not very different from the geometric representation of an algebraic polynomial. This follows from the fact that the function $\mathfrak{R}$ in the considered interval can be represented by the Taylor formula with a small error term. Therefore, the interpolation of the function by an algebraic polynomial should give good accuracy by taking a sufficient number of sites close to the point of interpolation $t$.

Construct an algebraic polynomial $P_{n}(t)$ of the degree $n$ satisfying the conditions

$$
P_{n}^{(a)}\left(t_{j}\right)=\Re^{(a)}\left(t_{j}\right), \quad \alpha=0,1, \quad j=1,2, \ldots, N
$$

We prove that there is only one interpolation polynomial $P_{n}(t)$ satisfying conditions (4). The interpolation conditions (4) represent a system of linear algebraic equations for the coefficients $a_{0}, a_{1}, \ldots, a_{n}$ of the polynomial

$$
P_{n}(t)=a_{0} t^{n}+a_{1} t^{n-1}+\ldots+a_{n} .
$$

The number of equations of this system is equal to the number of unknowns and is equal to $2 N$. Therefore, it suffices to show that the homogeneous system

$$
P_{n}^{(a)}\left(t_{j}\right)=0, \quad \alpha=0,1, \quad j=1,2, \ldots, N,
$$

has only the trivial solution $a_{0}=a_{1}=\ldots=a_{n}=0$. The condition (6) for a fixed $j$ and $\alpha=0,1$ means that the number $t_{j}$ is a root of the multiplicity $n$ of the polynomial $P_{n}(t)$. Thus, the polynomial $P_{n}(t)$ has just no less than $n+1$ roots on [a, b]. Since the degree of $P_{n}(t)$ is equal to $n$, this polynomial is identically zero, all its coefficients are equal to zero, the homogeneous system of equations (6) has a unique solution $a_{0}=a_{1}=\ldots=a_{n}=0$, and there is only one polynomial (5) satisfying the conditions (4).

The heterogeneous system (4) is uniquely solvable for any right-hand side. Since the values of $\mathfrak{R}^{(a)}\left(t_{j}\right), \alpha=0,1, \quad j=1,2, \ldots, N$ includes only the right side of system (4), the coefficients $a_{j}$ of the polynomial $P_{n}(t)$ are expressed linearly through the value of $\Re^{(a)}\left(t_{j}\right)$, i.e.,

$$
P_{n}(t)=\sum_{j=1}^{N} L_{j}(t) \Re^{\prime}\left(t_{j}\right) .
$$

We now find explicit representation of formula (7) with the help of the residual

$$
r_{n}(\Re, t)=\Re(t)-P_{n}(t) .
$$

It occurs, when replacing the function $\Re(t)$ with the interpolation polynomial $P_{n}(t)$, and is called the error of interpolation or residual member of the interpolation formula. Obviously, this error is zero at the nodes of the interpolation. We now estimate the error at an arbitrary point $t \in[a, b]$. 
Theorem 1. Let nodes $t_{j}(j=1,2, \ldots, N)$ and a point $t$ belong to the segment $[a, b]$, and let the function $\Re(t)$ have a continuous derivative of order $n+1$ on $[a, b]$. Then there exists a point $\xi$ on the segment $[a, b]$ such that the following equality holds for the interpolation error $r_{n}(\Re, t)$ :

$$
r_{n}(\Re, t)=\frac{\Psi_{n}(t)}{(n+1) !} \Re^{(n+1)}(\xi), \quad \Psi_{n}(t)=\prod_{j=1}^{N}\left(t-t_{j}\right)^{2} .
$$

Proof. Consider the auxiliary function

$$
\varrho(s)=\Re(s)-P_{n}(s)-K \Psi_{n}(s),
$$

where $s \in[a, b], K$ is a constant. Let us evaluate $r_{n}(\Re, t)$ at a given point $t \in[a, b]$, which is not a node of interpolation. We choose the constant $K$ from the condition $\varrho(s)=0$. It is enough to put

$$
K=\frac{\Re(t)-P_{n}(t)}{\Psi_{n}(t)} .
$$

The function $\varrho(s)$ has at least $n+2$ zeros on $[a, b]$, namely, at the points $t$ and $t_{j}(j=1,2, \ldots, N)$. The derivative of the first order $\varrho^{\prime}(s)$ by the theorem of Rolle has at least one zero within each interval between adjacent points $t_{1}, t_{2}, \ldots, t_{N}, t$. The number of zeros will be $N$. Moreover, $\varrho^{\prime}(s)$ has $n$ roots of the multiplicity one at each point $t_{j}(j=1,2, \ldots, N)$. Thus, $\varrho^{\prime}(s)$ has, given the multiplicity, of at least $n+1$ zeros on $[a, b]$. Similarly, the second-order derivative $\varrho^{\prime \prime}(s)$ has at least $n$ roots, etc. The derivative $\varrho^{(n+1)}(s)$ vanishes at least once on $[a, b]$. Thus, there exists a point $\xi \in[a, b]$, where $\varrho^{(n+1)}(s)=0$.

From (10), we have

$$
\varrho^{(n+1)}(s)=\mathfrak{R}^{(n+1)}-K_{n}(s) .
$$

Since $\Psi_{n}(s)$ is the polynomial of degree $n+1$ with leading coefficient 1, we have $\Psi_{n}(s)=$ $=(n+1)$ !. Therefore, in view of the condition $\varrho^{(n+1)}(s)=0$ and the expression for $K$, we obtain the following representation for the error of interpolation:

$$
\Re(t)-P_{n}(t)=\frac{\Psi_{n}(s)}{(n+1) !} \Re^{(n+1)}(\xi) .
$$

From this, it follows that (9) holds.

Without loss of generality, assume that the function $\mathfrak{R}(t)$ is analytic in the closed end area of $\mathfrak{H}$ bounded by $\wp$ and containing $[a, b]$. Then the Cauchy formula

$$
\Re(t)=\frac{1}{2 \pi i} \int_{\wp} \frac{\Re(t)}{\xi-t} d \xi
$$

allows us to reduce the construction of an interpolation polynomial $P_{n}(t)$ to the problem of finding a polynomial $P_{n}(1 /(\xi-t), t)$ for a fairly simple linear fractional functions $1 /(\xi-t)$, where $\xi$ is taken as a parameter over which the integration is carried out in (11). 
We investigate the dependence of the error of interpolation

$$
r_{n}\left(\frac{1}{\xi-t}, t\right)=\frac{1}{\xi-t} P_{n}\left(\frac{1}{\xi-t}, t\right)=\frac{1}{\xi-t}-\sum_{j=1}^{N} L_{j}(t) \frac{1}{(\xi-t)^{2}}
$$

on $\xi$. Equation (12) is an expansion of a rational function into simple fractions. It is obvious that the point $\xi=t$ for the error is a first-order pole with thr residue equal to one. The common denominator of all the terms in the expression for error (12) is equal to $(\xi-t) \Psi_{n}(\xi)$. Thus, the error $r_{n}(1 /(\xi-t), t)$ can be represented as

$$
r_{n}\left(\frac{1}{\xi-t}, t\right)=\frac{\Omega(\xi, t)}{(\xi-t) \Psi_{n}(\xi)},
$$

where $\Omega(\xi, t)$ is a polynomial in $\xi$ of degree not higher than $n-1$.

The polynomial $\Omega(\xi, t)$ on the right side of (13) does not depend on $\xi$ and is equal to $\Psi_{n}(\xi)$. Indeed, when $|\xi| \gg|t|$, the following equality holds:

$$
\frac{1}{\xi-t}=\sum_{I=1}^{\infty} \frac{t^{I}}{\xi^{I+1}} .
$$

It follows from (13) that the error is linearly dependent on the interpolated function. Consequently, we have

$$
r_{n}\left(\frac{1}{\xi-t}, t\right)=\sum_{I=1}^{\infty} \frac{r_{n}\left(t^{I}, t\right)}{\xi^{I+1}} .
$$

Since the functions $\varphi=t^{k}(k=0,1, \ldots, n)$ are interpolated exactly, we have

$$
r_{n}\left(t^{I}, t\right)=0, \quad I=0,1, \ldots, n .
$$

Thus, the first $n+1$ terms in sum (14) disappear, and expansion (14) begins with a member $r_{n}\left(s^{I+1}, s\right) / \xi^{I+2}$. It follows that the degree of the numerator $\Omega(\xi, s)$ in $(13)$ in $\xi$ is by $n+2$ units less than that of the denominator. But the degree of the denominator is equal to $n+2$. Thus, $\Omega(\xi, s)$ is of zero degree and does not depend on $\xi$, i.e., $\Omega(\xi, t)=\Omega(t)$.

Finally, since the value $\xi=t$ for $r_{n}(1 /(\xi-t), t)$ is a simple pole, minus 1 , we have $\Omega(t)=\Psi_{n}(t)$ and

$$
r_{n}\left(\frac{1}{\xi-t}, t\right)=\frac{\Psi_{n}(t)}{(\xi-t) \Psi_{n}(\xi)} .
$$

Taking (11) - (15) into account, we obtain a representation of error $r_{n}(\Re, t)$ in the form of a contour integral

$$
r_{n}(\Re, t)=\frac{1}{2 \pi i} \int_{\wp} \Re(\xi) r_{n}\left(\frac{1}{\xi-t}, t\right) d \xi=\frac{\Psi_{n}(t)}{2 \pi i} \int_{\wp} \frac{\Re(\xi)}{(\xi-t) \Psi_{n}(\xi)} d \xi .
$$


The resulting integral expression is the basis for finding the required interpolation polynomial

$$
P_{n}(t)=\Re(t)-r_{n}(\Re, t) .
$$

The residue function $\Psi_{n}(t) \Re(\xi) /\left[(\xi-t) \Psi_{n}(\xi)\right]$ at the point $\xi=t$ is, obviously, $\Re(t)$. Calculate the residue at the pole $\xi=t_{j}, j=1,2, \ldots, N$. For values of $\xi$ close to the values of $t_{j}$, the following expansions in power series hold:

$$
\begin{aligned}
& \Re(\xi)=\sum_{I=0}^{\infty} \frac{1}{I !} \mathfrak{R}^{(I)}\left(t_{j}\right)\left(\xi-t_{j}\right)^{I}, \\
& \frac{1}{\xi-t}=-\frac{1}{\left(t-t_{j}\right)-\left(\xi-t_{j}\right)}=\sum_{I=0}^{\infty} \frac{(\xi-t)^{I}}{\left(t-t_{j}\right)^{I+1}}, \\
& \frac{\xi-t_{j}}{\Psi_{n}(\xi)}=\sum_{I=0}^{\infty} C_{I}^{(j)}\left(\xi-t_{j}\right)^{I} .
\end{aligned}
$$

Representing the function $\Re(\xi) /\left[(\xi-t) \Psi_{n}(\xi)\right]$ in the form of:

$$
\frac{\Re(\xi)}{(\xi-t) \Psi_{n}(\xi)}=\frac{1}{\xi-t_{j}} \cdot \frac{\xi-t_{j}}{\Psi_{n}(\xi)} \cdot \frac{\Re(\xi)}{\xi-t},
$$

find the rest of it by multiplying the power series (18).

Expressions (17), (18), and (19) and the equality $\mathfrak{R}^{\prime}\left(t_{j}\right)=F\left(t_{j}\right)$ according to (1) lead to the desired interpolation polynomial

$$
\begin{aligned}
& P_{n}(t)=\sum_{j=1}^{N} \frac{\aleph^{2}(t)}{\left(t-t_{j}\right)^{2}\left[\aleph^{\prime}\left(t_{j}\right)\right]^{2}} \times \\
& \times\left\{\left[1-\frac{\boldsymbol{\aleph}^{\prime \prime}\left(t_{j}\right)}{\boldsymbol{\aleph}^{\prime}\left(t_{j}\right)}\left(t-t_{j}\right)\right] \Re\left(t_{j}\right)+\left(t-t_{j}\right) F\left(t_{j}\right)\right\},
\end{aligned}
$$

where

$$
\boldsymbol{\aleph}(t)=\sqrt{\Psi_{n}(t)}=\prod_{j=1}^{N}\left(t-t_{j}\right) .
$$

Above, we assumed that the function $\mathfrak{R}(t)$ is analytic in the closed end area of $\mathfrak{H}$, bounded by $\wp$ and containing $[a, b]$. But (20) contains only the values $\mathfrak{R}^{(a)}\left(t_{j}\right), j=1,2, \ldots, N, \alpha=0,1$. Thus, formula (20) remains true for any function $\Re(t)$ with finite values of $\Re^{(a)}\left(t_{j}\right), j=1,2, \ldots, N$, $\alpha=0,1$.

The interpolation polynomial (20) for $N=3$ and $\forall t \in\left[t_{j-1}, t_{j}\right]$ is simplified to a polynomial of the fifth degree: 


$$
\begin{aligned}
& P_{3}(t)=\left(\frac{t_{j}-t}{t_{j}-t_{j-1}}\right)^{2}\left(\frac{t-t_{j-1}}{t_{j}-t_{j-1}}\right)^{2}\left[\left(1+\frac{3}{4} \frac{t-t_{j-1}}{t_{j}-t_{j-1}}\right) \Re_{j-2}+\frac{1}{4}\left(t-t_{j-2}\right) F_{j-2}\right]+ \\
& +\left(\frac{t_{j}-t}{t_{j}-t_{j-1}}\right)^{2}\left(\frac{t-t_{j-2}}{t_{j-1}-t_{j-2}}\right)^{2}\left[\Re_{j-1}+\left(t-t_{j-1}\right) F_{j-1}\right]+ \\
& +\left(\frac{t-t_{j-2}}{t_{j-1}-t_{j-2}}\right)^{2}\left(\frac{t-t_{j-1}}{t_{j}-t_{j-1}}\right)^{2}\left[\left(1-\frac{3}{4} \frac{t-t_{j-1}}{t_{j}-t_{j-1}}\right) \Re_{j}-\frac{1}{4}\left(t_{j}-t\right) F_{j}\right] .
\end{aligned}
$$

Finally, when $N=2$ and $\forall t \in\left[t_{j-1}, t_{j}\right]$, we have the third-degree polynomial

$$
\begin{aligned}
& P_{2}(t)=\left(\frac{t_{j}-t}{t_{j}-t_{j-1}}\right)^{2}\left[\left(1+2 \frac{t-t_{j-1}}{t_{j}-t_{j-1}}\right) \Re_{j-1}+\left(t-t_{j-1}\right) F_{j-1}\right]+ \\
& +\left(\frac{t-t_{j-1}}{t_{j}-t_{j-1}}\right)^{2}\left[\left(1+2 \frac{t_{j}-t}{t_{j}-t_{j-1}}\right) \Re_{j}+\left(t_{j}-t\right) F_{j}\right] .
\end{aligned}
$$

One can make sure that the last formula gives correct results at $\mathfrak{R}=1, t, t^{2}, t^{3}$. At $\mathfrak{R}=t^{4}$, the maximal error $r_{\max }$ in the range $(0,1)$ is $r_{\max }=-0,0625$. At the same time, by the Bessel formula, $r_{\text {max }}=-0.5625$ with the same number of given $\Re\left(t_{j}\right)$ values.

We indicate the main advantages of these interpolation formulas built on the basis of predetermined values of the function and its derivative $\Re^{(a)}\left(t_{j}\right), j=1,2, \ldots, N, \alpha=0,1$ :

they are more accurate than any of the formulas that use only the value of $\Re\left(t_{j}\right)$;

when interpolating inside the interval $\left(t_{N-1}, t_{N}\right)$ we do not need the data outside of the right border of the segment of interpolation, so they can be used for the rightmost interval;

values of the function $\mathfrak{R}\left(t_{j}\right)$ and its derivative $\mathfrak{R}^{\prime}\left(t_{j}\right)$ can be set on an irregular grid $t_{j}$.

Conclusion. The results obtained in this paper indicate that solving problems of the type (1), (2) for ordinary differential equations can be carried out effectively by the interpolation (the methodology is developed in [7]). Here, we give an outlook of numerical methods developed by the authors for effective regional solutions of boundary-value problems with a prehistory on the basis of the "one-way interaction" approach. Within this approach, we construct our own method for filling the data, given on the macroscale grid nodes, in the mesoscale network based on the spline interpolation and a precise (the fourth order of accuracy) numerical method for the approximation of the first- and second-order derivatives in differential equations. The method is implemented within the paradigm of the formalized design of parallel programs [8].

\section{REFERENCES}

1. Asselin, R. (1972). Integration of a semi-implicit model with time-dependent boundary conditions. Atmosphere, 10, pp. 44-55.

2. Davies, H. C. (1976). A lateral boundary formulation for multi-level prediction models. Quart. J. Roy. Meteorol. Soc., 102, pp. 405-418. 
3. Doroshenko, A. Yu. \& Prusov, V. A. (2005). Methods of efficient modeling and forecasting regional atmospheric processes. In Advances in Air Pollution Modeling for Environmental Security (pp. 143-152). NATO Science Series, Vol. 54. Dordrecht: Springer.

4. Prusov, V. A. \& Doroshenko, A. Yu. (2006). Modeling natural and technogenic atmospheric processes. Kyiv: Naukova Dumka (in Ukrainian).

5. Miyakoda, K. \& Rosati, A. (1977). One-way nested grid models: The interface condition and the numerical accuracy. Mon. Weather. Rev., 105, pp. 1092-1107.

6. Manabe, S. (Ed.). (1985). Issues in atmospheric and oceanic modeling. Part A and Part B. Orlando: Academic Press.

7. Prusov, V. \& Doroshenko, A. (2018). Computational techniques for modeling atmospheric processes. Hershey, PA, USA: IGI Global.

8. Doroshenko, A., Ivanenko, P., Ovdii, O. \& Yatsenko, O. (2016). Automated program design - an example solving a weather forecasting problem. Open Physics, 14, Iss. 1, pp. 410-419. https://doi.org/10.1515/phys-20160048

Received 12.12.2019

\title{
В.А. Прусов ${ }^{1}$, А.Ю. Дорошенко ${ }^{2}$
}

${ }^{1}$ Український гідрометеорологічний інститут ДСНС України та НАН України, Київ

${ }^{2}$ Інститут програмних систем НАН України, Київ

E-mail: doroshenkoanatoliy2@gmail.com

ЕФЕКТИВНИЙ ОБЧИСЛЮВАЛЬНИЙ МЕТОД ДЛЯ МЕЗОМАСШТАБНОГО ПРОГНОЗУВАННЯ ПОГОДИ

Розглянуто математичні задачі, пов'язані з чисельним розв'язуванням рівнянь для прогнозних моделей регіональної циркуляції атмосфери. Описано методологію ефективного регіонального вирішення крайових задач з передісторією на основі підходу “однобічної взаємодії”. У рамках цього підходу запропоновано метод заповнення даних з вузлів макромасштабної сітки у мезомасштабну сітку на основі інтерполяції сплайном і точного чисельного методу (четвертого порядку точності) для наближення похідних першого та другого порядку диференціальних рівнянь. Тим самим розв’язування задач для звичайних диференціальних рівнянь може ефективно здійснюватися шляхом інтерполяції.

Ключові слова: мезомасштабне прогнозування погоди, диференціальні рівняння, інтерполяція.

\author{
В.А. Прусов ${ }^{1}$, А.Е. Дорошенко ${ }^{2}$ \\ ${ }^{1}$ Украинский гидрометеорологический институт ГСЧС Украины и НАН Украины, Киев \\ ${ }^{2}$ Институт программных систем НАН Украины, Киев \\ E-mail: doroshenkoanatoliy2@gmail.com
}

\section{ЭФФЕКТИВНЫЙ ВЫЧИСЛИТЕЛЬНЫЙ МЕТОД} ДЛЯ МЕЗОМАСШТАБНОГО ПРОГНОЗИРОВАНИЯ ПОГОДЫ

Рассмотрены математические задачи, связанные с численным решением уравнений для прогнозных моделей региональной циркуляции атмосферы. Описана методология эффективного регионального решения краевых задач с предысторией на основе подхода “одностороннего взаимодействия”. В рамках этого подхода предложен метод заполнения данных с узлов макромасштабной сетки в мезомасштабную сетку на основе интерполяции сплайном и точного численного метода (четвертого порядка точности) для приближения производных первого и второго порядка дифференциальных уравнений. Тем самым решение задач для обыкновенных дифференциальных уравнений может эффективно осуществляться путем интерполяции.

Ключевые слова: мезомасштабное прогнозирование погоды, дифференциальные уравнения, интерполяция. 